\title{
Building a 3D faulted a priori model for stratigraphic inversion: illustration of a new methodology applied on a North Sea field case study
}

Jean-François Rainaud, Vincent Clochard, Nicolas Delépine, Thomas Crabié (IFP Energies nouvelles) Mathieu Poudret, Michel Perrin (GEOSIRIS), and Emmanuel Klein (IFP Energies nouvelles, currently EXTIA).

\section{Corresponding author : jean-francois.rainaud@ifpen.fr}

\begin{abstract}
Accurate reservoir characterization is needed all along the development of an oil and gas field study. It helps building 3D numerical reservoir simulation models for estimating the original oil and gas volumes in place and for simulating fluid flow behaviors. At a later stage of the field development, reservoir characterization can also help deciding which recovery techniques need to be used for fluids extraction.
\end{abstract}

In complex media, such as faulted reservoirs, flow behavior predictions within volumes close to faults can be a very challenging issue. During the development plan, it is necessary to determine which types of communication exist between faults or which potential barriers exist for fluid flows. The solving of these issues rests on accurate fault characterization. In most cases, faults are not preserved along reservoir characterization workflows. The memory of the interpreted faults from seismic is not kept during seismic inversion and further interpretation of the result. The goal of our study is at first to integrate a 3D fault network as a priori information into a model-based stratigraphic inversion procedure. Secondly, we apply our methodology on a well-known oil and gas case study over a typical North Sea field (UK Northern North Sea) in order to demonstrate its added value for determining reservoir properties. More precisely, the a priori model is composed of several geological units populated by physical attributes, they are extrapolated from well log data following the deposition mode, but usually a priori model building methods respect neither the 3D fault geometry nor the stratification dips on the fault sides. We address this difficulty by applying an efficient flattening method for each stratigraphic unit in our workflow. Even before seismic inversion, the obtained stratigraphic model has been directly used to model synthetic seismic on our case study. Comparisons between synthetic seismic obtained from our 3D fault network model give much lower residuals than 
with a "basic" stratigraphic model. Finally, we apply our model-based inversion considering both faulted and non-faulted a priori models. By comparing the rock impedances results obtain in the two cases, we can see a better delineation of the Brent-reservoir compartments by using the 3D faulted $a$ priori model built with our method.

\section{Introduction}

Stratigraphic inversion is a nice way for integrating various kinds of data at various scales such as seismic and well log data. The inversion process itself computes an optimal earth model, usually parameterized by acoustic/elastic parameters like $P$ - and $S$-wave impedances. These optimal attributes can be used to deduce geo-mechanical parameters like Poisson's ratio and Young's modulus to improve field development. Many inversion techniques are available but integrating a 3D fault network in the inversion process still remains a challenging task. In faulted areas, the classical methods used for building an a priori model do not respect the 3D fault geometry, since the fault throws are not taken into account. In a "basic" model building, for each interpreted seismic horizon with a discontinuity caused by a fault, the part of the fault limited by its horizon intersection is integrated and smoothed as a continuous part of the horizon itself. It is a rather good approximation to define limits of the different geological units. But the approximation is going to be false to define sedimentary deposit mode close to the faults (double arrow on top of Figure 1). Otherwise, to avoid the time consuming task of fault/horizon interpretation, Hale $(2009,2010)$ proposed an image-guided interpolation method to compute a subsurface model that conforms to both seismic structures and well-log measurements. A review of the various used methods is summarized in Hoyes and Cheret (2011). Hale (2013) also proposes a method of automatic fault-picking. This method was recently improved by a supervised machine learning algorithm (Guitton et al., 2017). More sophisticated methods, based on the use of structure tensors estimate the orientations of the structural and stratigraphic features (Wu, 2017). Specific difficulties like unconformities, which generate hiatus in the Wheeler domain (Wheeler, 1958) are also addressed by Wu and Hale (2015). 
In spite of their interest, all of these methods are sensitive to weak seismic amplitudes close to the faults. This is the main reason why automatic interpretation of horizons and faults based on cognitive vision was proposed as an alternative solution (Verney et al., 2008).

We will consider here the case when a set of interpreted horizons and a 3D fault network are available. The methodology that we propose addresses the difficulties due to the presence of unconformities like on-laps and erosive surfaces.

The first part of our study describes a three-step workflow, which integrates the 3D fault network by means of the a priori model, used by the inversion process.

The second part of our work is an application of our workflow on a real case study provided by TOTAL to the Energistics RESQML members for testing purpose. These data are extracted from a real reservoir dataset located in the UK Northern North Sea (Alwyn North field): a faulted and nonfaulted have been built by extrapolating rock impedances from well log data. Then we intend to evaluate the added value brought by stratigraphic inversion using a faulted a priori model. The results provided by our methodology are compared to those provided by an inversion workflow based on a non-faulted a priori model that we performed for comparison.

\section{Methodology of 3D faulted a priori model building}

Reconstructing geology before fault activation is a key step in the methodology: efficient flattening method, such as Borouchaki (2008) has been used here. It allows extracting and unfolding a stratigraphic unit for updating the property repartitions (Horna et al., 2010) or realize a global transformation (Dulac, 2009). J.C Dulac uses the global uvt-transform method, proposed by Mallet $(2004,2014)$, which is a general space-time mathematical framework for the domain transform. This method has been applied to remove folding and faulting (Labrunye et al., 2009; Mallet et al., 2010) in a seismic image. The originality of our approach consists in unfolding independently each stratigraphic unit sequence and operates the final reconstruction of the a priori model by respecting geological rules defined in Perrin (1998). 
In many cases, uncertainties regarding the contact localization between the picked seismic horizons and faults can produce surface modelling artefacts due to a lack of information. We set some toolbased geological rules to characterize the horizon-to-fault contacts, which help to topologically associate the two sides of a horizon separated by a fault. Thus for each horizon, the fault gaps are topologically identified. In our case a topological consistent model, the Chrono-tops, Chrono-bottoms and unconformities are extended in the whole seismic bloc limits. This method allows to add more interpretative constraints than an automatic analysis of the information captured in a seismic bloc, specifically on complex area. These surface extrapolations can complement a preliminary manual or automatic seismic interpretation of horizon and faults (Verney, 2008) or other methods for fault characterization.

In the following, we consider a picked conformable horizon modified by tectonics, so it can be flattened once the fault gaps have been removed by using the flattening method defined in Rainaud et al. (2015). But in some cases, the picked horizon cannot be considered as an isochron surface everywhere; this may happen in the cases of erosion, onlap or intrusive geobodies. In such cases, we perform a preliminary complementary characterization and modelling of the more important stratigraphic limits in order to complete the structural information, even in the areas where the information no longer exists in the today geology.

In some situations, an interpreted seismic horizon can correspond to an erosion or a deposition surface. An illustration of erosion mode on our case study will be presented in the following.

After adding the above constraints, we implemented the following three-step workflow for building the faulted a priori model starting from horizons, unconformities and faults pickings:

(1) The topological modelling of the stratigraphic unit boundaries is based on their relative geological age. Each topological model (for each stratigraphic boundary and picked unconformity) includes a set of fault gap connections, which result from the intersection of a fault network with the stratigraphic interfaces. A 3D-sealed surface framework is then exported (Figure 3).

(2) Each geological unit is flattened and transported into its original depositional $(U, V, D)$ domain. We operate an isometric unfolding based on the minimization of the elastic tensor deformation (Poudret et al., 2012 and Bennis et al., 2012). To achieve this process, geological units are meshed 
by hexahedrons in the $(U, V, D)$ domain according to fault geometry and these hexahedrons are replaced in the present day seismic reference domain $(X, Y, T$ woWayTime $)$ or $(X, Y$, Depth $)$ according to seismic imaging domain (Figure 4).

This guides us for building, a flat volume in the depositional $(U, V, D)$ domain for each stratigraphic unit. This volume can be enveloped in a one cell thick Cartesian grid (Figure 5, top). This grid, is easy to build since it roughly respects the InLine, XLine and a vertical seismic sampling. We additionally use the pillars of the Cartesian grid for defining a hexahedral mesh along the fault network (Figure 5, bottom). A particularity of our method consists in using these two meshes together, even if they are not totally coincident. Consequently, for each hexahedral cell of both meshes, we have an image function in the $(U, V, D)$ domain and its corresponding image in the seismic domain in order to fill the faulted a priori model.

At the end, we can associate a seismic pixel location to one node of the corresponding hexahedron defined in the $(U, V, D)$ domain. We are then able to transfer information between the two domains. The transfer mechanism is based on shape transform functions that estimate the parametric position of a point in a hexahedron (Dhatt et al., 2001). These shape functions define a natural coordinate system whose origin is located at the center of the hexahedron (Figure 6). The shape functions are used to interpolate the coordinates from the nodal coordinates and we can then transfer the property information associated to any point from the $(X, Y$,Time or Depth) domain to the $(U, V, D)$ domain. For overcoming the fact that hexahedron meshes built on the base of a regular Cartesian grid and along faults are not totally coincident, we use a location quality criteria that allows us to choose which cells must be prioritized when overlapping conflicts occur between the Cartesian and the fault conformable meshes. Figure 6 illustrates the 2D shape functions and the quality criteria that we use for deciding which of two concurrent hexahedrons can be affected to a given point $\mathrm{P}$. The global parametric estimation is 1 in the center of the hexahedron.

(3) Using the same mechanism of transformation from the seismic to the depositional $(U, V, D)$ domains, we also transfer the well trajectories and their log information to being able to extrapolate them by using determinist and/or geostatistical algorithms. The Cartesian grid is then 
filled with properties according to the mode of deposition (Rainaud et al., 2015). The computed properties are transferred back to the seismic domain in order to be used in a stratigraphic inversion software.

The whole three-step workflow can be applied indifferently in Depth or Two Way Time (TWT) domains.

\section{Methodology of stratigraphic inversion}

Our stratigraphic inversion methodology is model-based. It uses post-stack and/or pre-stack seismic data in the time domain, and more precisely, data are obtained by performing partial stacks according to several ranges of incidence angles at the reservoir level. The model integrates geological and geophysical information in a structural framework defined by interpreted seismic horizons. It also includes the sediment deposition modes within each geological unit. The inversion problem itself is solved by a conjugate gradient algorithm, which requires an initial model equal to the a priori model. This model is constructed by extrapolating $P$ - and $S$-wave impedances computed from well log data. The extrapolation follows the stratigraphy defined inside each geological unit of the geometrical framework. The use of pre-stack data allows us to invert for an elastic model parameterized by $P$ - and $S$-wave impedances (Clochard et al., 2009) whereas post-stack inversion yields only one parameter, namely, the acoustic impedance (Delépine et al., 2009). The stratigraphic inversion workflow is divided in three steps:

a. Well-to-seismic calibration

The main objective of this step is to define, for each angle class, an optimal wavelet, which relates the well log data to the seismic data and compensates for uncertainties due to pre-processing issues such as surface static corrections, source deconvolution, etc.

The well-to-seismic calibration procedure involves the repetitive computation of a synthetic trace at the well location for each angle class. The synthetic seismograms are calculated via time convolution of a trial wavelet with the reflection coefficient series obtained from the well log elastic impedances and density values constructed from the seismic velocity and density values obtained by well logging. The dependency of the reflection coefficient as a function of incident angle given by the linearized 
expressions of Aki and Richards (1980). One can note that only one wavelet is computed with poststack data

b. A priori model building without faults

The interpreted horizons picked from seismic data provide the structural framework of the model with its main geological units. We then consider the mode of sedimentary deposition inside each geological units (// to Top, // to Bottom, Proportional) for computing correlation surfaces, also used for interpolating various kinds of properties (physical like elastic impedances, density or geometric like local dip) between the wells retained in the calibration step (1). Well log data are further used to provide the low frequency components of the a priori model not contained in the seismic data. One can note with post-stack data, only the physical attribute called acoustic impedance is used. With prestack data we have a maximum of 3 elastic earth parameters like $P$ - and $S$-wave impedances and density.

c. Joint pre-stack stratigraphic inversion of all angle classes

Our pre-stack stratigraphic inversion algorithm is based on a Bayesian approach. The specific algorithm employed in this study was described by Tonellot et al. (2001): the model-based inversion optimizes an earth model $m$ parameterized by 3 elastic parameters called $P$-wave impedance $\left(I_{P}\right), S$ wave impedance $\left(I_{S}\right)$, and density $(\rho)$, defined for each pixel of the interest area (Figure 1). The functional $F$ to be minimized is a summation of two terms:

$F(m)=F_{\text {seis }}(m)+F_{\text {geol }}(m)$

with :

$F_{\text {seis }}(m)=\frac{\left\|S(m)-S^{o b s}\right\|^{2}}{\sigma_{S}^{2}}$

$F_{\text {seis }}$ is called the seismic term because it depends on the current model $m$ and on the observed seismic data $S^{o b s} . \sigma_{S}$ is the uncertainty about observed seismic data $S^{o b s}$. The measured signal to noise ratio $S N R$ is converted into $\sigma_{S}$ via the formula:

$$
\sigma_{S}=s_{R M S} / S N R
$$

$S_{R M S}$ being the RMS value of observed seismic data $S^{\text {obs }}$. 
$F_{\text {geol }}(m)=F_{\text {geoll }}(m)+F_{\text {geol } 2}(m)=\frac{\left\|m-m^{\text {prio }}\right\|^{2}}{\lambda \sigma_{G}^{2}}+\frac{\lambda\left\|P_{T}\left(\nabla\left(m-m^{\text {prio }}\right)\right)\right\|^{2}}{\sigma_{G}^{2}}$

$F_{\text {geol }}$ is called the geological term because it depends on the a priori geological model and on the current model $m$ to compute the synthetic seismic data $\sigma_{G}$ is the uncertainty about the a priori model $m^{p r i o}$, and $\lambda$ is the correlation length of this uncertainty. In the right term, $P_{T}$ projects the gradient of $I_{P}$, $I_{S}$ and $\rho$ on the plane that is tangent to the local leaf of the stratigraphic foliation. Minimizing this term makes the lateral variations of $I_{P}, I_{S}$ and $\rho$ similar to those of the a priori model.

From the above explanations and looking at the Figure 1, one can understand how the deposit mode apart from each side of the fault could influence the cost function through the local dip of the layering.

\section{Application to a real case study: the Alwyn North field (North Sea)}

a) Structural model of Alwyn with faults

The Alwyn North field is a prospect located in the North Sea basin (see figure 7). The major steps of the geological evolution of this part of the North Sea basin are the following:

1. Permo-Trias: ante-Permian deposits are subject to an EW extension inducing NS and EW trending faults; there occurs a basin subsidence towards East and a deposition of the Cormorant et Statfjord formations.

2. Lower Jurassic : deposition of the Dunlin formation in a period of tectonic quietness.

3. Middle Jurassic, new extension inducing a reactivation of the existing faults; deposition of the Brent formation.

4. Callovo-Oxfordian / Kimmeridgian stages: deposition of the Heather formation and of the Kimmeridge Clays; continuous tectonic movements induce a tilting of the faulted blocks; these movements cease before the end of the Kimmeridge Clays deposition.

5. Late Jurassic: deposition of the Cromer formation; an erosion period follows, which induces the disappearance of the parts of Cromer and Humber formations that were located on the crest zones; this erosion is signalled by a surface called BCU (Base Cretaceous Unconfomity). 
6. Lower Cretaceous: deposition of Lower Turonian sediments over the BCU erosion surface.

7. Upper Cretaceous / Tertiary: deposition of the Shetland Group sediments that have no interest for oil prospecting.

The structural style of the terrains located below the BCU is characterized by geological blocks eroded and tilted towards West. These blocks are aligned along a North South direction parallel to the major faults that cross the Alwyn field. This constitutes a major control for petroleum trapping.

On figures 2 and 7, we can see that bottom of Kimmeridge Clays formation erodes the Heather formation.

In this case, we can use a geological rule for generating the missing parts of the chronological top of the Heather unit (complementary purple horizon added on Figure 2, part B). It is thus possible to flatten the Chrono Top (purple horizon) and the Chrono Bottom (green horizon) of the Heather unit in order to reconstruct its original deposition environment. By adding this "synthetic" information, we separately constrain each stratigraphic unit. We can then construct a full 3D faulted a priori model by applying geological based priority rules. The three pictures of the Figure 2 illustrate the extrapolation operated for flattening Chrono Tops by using geological rules. This extrapolation is operated in order to add necessary constraints during the modeling process but these constraints are not reused in the final step when considering the erosion process that involves the bottom of Kimmeridge Clays and the Base Cretaceous Unconformity (BCU).

b) Seismic inversion of Alwyn using 3D faulted model

Before inversion, synthetic seismic have been modeled using the faulted and non-faulted acoustic impedance models (Figure 8, bottom). Using real seismic data, residuals have been estimated. The residual estimation corresponds to the initial step of the inversion, the computation of the seismic term only : As a result, a much lower value (50\% less) of the cost function is observed using the faulted $a$ priori model. It is an important result which confirms that fully taking into account the 3D fault network as a priori information gives a better acoustic impedance model and corresponding synthetic seismic closer to real seismic. 
The two impedance models have been used independently in two stratigraphic inversions as initial and a priori model. Intuitively one can understand having a closer initial model from the final solution will help the convergence in the inversion process.

The inversion parameters are the same in both cases. As a result, the reduction of the cost function has the same behavior and we have a good random distribution of the residuals compared to the observed seismic at the same scale (Figure 9, left and middle).

We have extracted horizontal slices rather than horizon maps, which are not accurate enough close to the fault network, at 3100 and 3130 meters depths, i.e. through the Brent formation (after Two Way Time to Depth conversion of the inversion results). Using the faulted a priori model, we can see lateral variations with enhanced impedance contrast for the whole Brent formation appearing in blue colors on Figure 10 (B and D). The low-impedance layer at the BCU level extends laterally with a better delineation on both sides of the fault (Figure 9, right). The geological block displayed on Figure 9 is also better characterize in term of impedance values.

\section{Conclusions}

We have described a full methodology taking into account a 3D fault network to build a 3D faulted $a$ priori model. It is then filled by various kinds of properties, physical like rock-impedances and geometric like local dip, at a seismic scale (Cartesian grid). This model is an assembly of stratigraphic units embedded in a faithful fault network. Different geological events such as erosion, like the one which generates the Base Cretaceous Unconformity, are taken into account.

During the process, we unfold the stratigraphic units separately respecting their modes of deposition. From the flattened deposition $(U, V, D)$ domain we are able to automatically build a mesh conform to fault network and interpreted horizons in order to establish a multidirectional transformation between this mesh and its image in the $(X, Y, T W T$ or $Z)$ domains, depending of input-seismic kinds Two Way Time (TWT) or Pre-Stack Depth Migration (PSDM). 
Then, by using 3D shape transform functions between these different domains, we can transfer well $\log$ information in the $(U, V, D)$ domain. We then extrapolate log data using deterministic and/or geostatistical algorithms to fill the hexahedron meshes. The computed transformation allows us to go back to the initial structural domain where cubes are filled at the seismic scale with physical properties like impedances and geometrical attributes like dips belonging to deposit mode.

For comparison purposes, we have built the non-faulted a priori model from the same series of interpreted horizons. Then we have used the faulted and non-faulted a priori models to run twice the stratigraphic inversion. The inversion result obtained with a faulted a priori model emphasize the delineation of the Brent reservoir compartmentalized by a fault network due to the extension of the North Sea basin. Further work will consist in improving the inversion process by taking into account variable inversion parameters, such as correlation length, according to the distance with the fault network.

\section{Acknowledgements}

This work was realized in the context of the demonstration of the exchange of structural and stratigraphic information between Energistics RESQML ${ }^{\mathrm{TM}}$ aware software. We would like to thank TOTAL which gave to Energistics Consortium members the detailed information and the data of former campaigns (e.g. 1998) concerning the UK Northern North Sea field, used to perform this use case (Morandini et al., 2011). TOTAL provided the initial interpreted horizons and faults embedded in a RESQML ${ }^{\mathrm{TM}}$ Energistics Package (Resqml V1 then V2 EPC documents). The communication between the independent software applications that we used along the workflow, was ensured through RESQMLTM EPC exchanges.

\section{References}

1. Aki, K. and Richards, P.G. [1980] Quantitative seismology - Theory and Methods. W.H. Freeman and Co., San Francisco.

2. Bennis, C., Borouchaki, H., Dumont, C., Lerat, O. and Rainaud, J.F [2012] 3D linesupport grid flattening for more accurate geostatistical reservoir population with 
petrophysical properties. Springer Verlag Engineering with Computers DOI 10.1007/s00366-012-0311-9.

3. Borouchaki, H. [2008] APLAT3D Surface Flattening Toolkit. University of Technology of Troyes.

4. Dhatt G., Touzot G. [2001] Méthode des éléments finis, ISBN 2-7637-6912-8 (presses de l'Université Laval).

5. Clochard, V., Delépine, N., Labat, K. and Ricarte, P. [2009] Poststack versus prestack stratigraphic inversion for $\mathrm{CO}_{2}$ monitoring purposes: A case study for the saline aquifer of the Sleipner Field. $79^{\text {th }}$ SEG Annual Meeting, Houston, USA, Expanded Abstracts, 24172421.

6. Dulac, J.C. [2009] Advances in chrono-stratigraphic interpretation modeling. First Break, vol. 27, page 69-72.

7. Guitton, A., Wang, H., Trainor-Guitton, W. [2017] Statistical imaging of faults in 3D seismic volumes using a machine learning approach. $87^{\text {th }}$ SEG Annual Meeting, Houston, USA, Expanded abstract, 2045-2049.

8. Hale, D. [2009] Structure-oriented smoothing and semblance: Center for Wave Phenomena, Report CWP-635.

9. Hale, D. [2010] Image-guided 3D interpolation of borehole data, $80^{\text {th }}$ SEG Annual International Meeting, Denver, USA., Expanded Abstracts, pp. 1266-1270.

10. Hale, D. [2013] Methods to compute fault images, ex-tract fault surfaces, and estimate fault throws from 3d seismic images: Geophysics, 78, no. 2, O33-O43, https://doi.org/10.1190/geo2012-0331.1.

11. Horna, S., Bennis, C., Crabié, T., Peltier, S., Rainaud, J.F., Poudret, M. [2010] Extracting and Unfolding a Stratigraphic Unit to update property population. EAGE and ECMOR.

12. Hoyes, J. and Cheret, T. [2011] A review of "global" interpretation methods for automated 3D horizon picking: The Leading Edge, 30, no. 1, 38-47, http://dx.doi.org/10.1190/1.3535431.

13. Morandini, M., Michel, B., Verney, P., Rainaud, J.F., Deny, L., Dulac, J.C., Fitzpatrick, T., Eastick, R.,Towery, L. [2011] Using RESQML ${ }^{\mathrm{TM}}$ for Shared Earth Model Data Exchanges between Commercial Modelling Applications and In-House Developments, Demonstrated on Actual Subsurface Data. Digital Energy Conference, Houston SPE143846.

14. Perrin, M. [1998] Geological consistency: an opportunity for safe surface assembly and quick model exploration. 3D Modeling of Natural Objects, A Challenge for the 2000's, $3(4-5)$. 
15. Poudret, M., Bennis, C., Dumont, C, Lerat, O., Rainaud, J.F. [2012] New flattening-based methodology for more accurate geostatistical reservoir populating. SPE/EUROPEC EAGE 2012.

16. Rainaud, J.F., Clochard, V., Crabié, T. and Borouchaki, H. [2015] Using a ChronoStratigraphic Unfolding Workflow to build an a priori model for Stratigraphic Inversion with accurate Horizon and Fault Fitting. SEG Annual Meeting, New Orleans, USA, Expanded Abstracts, 1927-1931

17. Tonellot, T., Macé, D. and Richard, V. [2001] Joint stratigraphic inversion of angle-limited stacks. $71^{\text {st }}$ SEG Annual Meeting, San Antonio, USA, Expanded Abstracts, 227-230.

18. Verney, P., Perrin, M., Thonnat, M. and Rainaud, J.,R. [2008] An approach of seismic interpretation based on cognitive vision: $70^{\text {th }}$ EAGE Conference and Exhibition. Rome, Italy.

19. Wheeler, H.,E. [1958]. Time stratigraphy: AAPG Bulletin, 42, 1047-1063

20. Wu, X. and Hale, D. [2015] 3D seismic image processing for unconformities. Geophysics. Vol.80, No.2

21. Wu, X. [2017] Structure-, stratigraphy- and fault-guided regularization in geophysical inversion. Geophysical Journal International, 210, 184-195.

22.

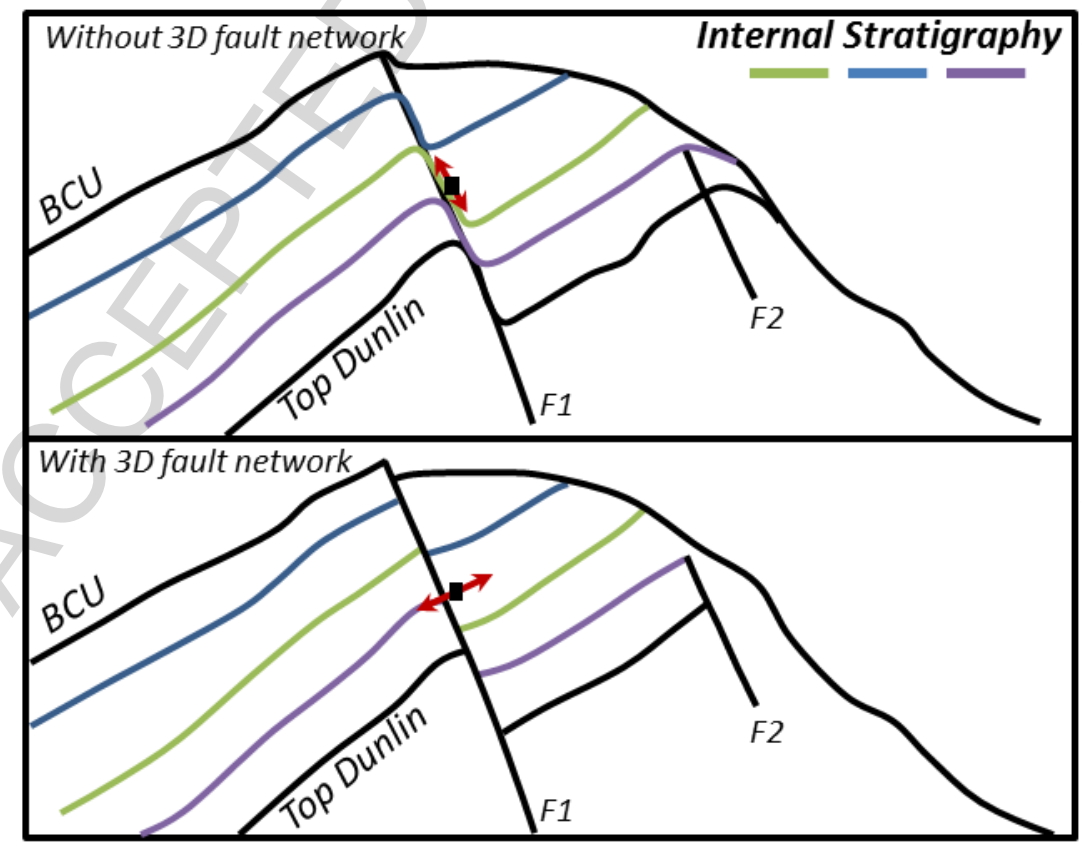

23.

24. Figure 1: Schematic view, inspired from North Sea Field, of internal stratigraphy built from interpreted horizon BCU and Top Dunlin. One can see strong dipping stratigraphy close to the fault when people apply horizon smoothing (see black pixel with red arrow, upper part). If $3 D$ 
fault network is taken into account dip of the stratigraphy is in agreement with mode of deposition defined by the user between BCU and Top Dunlin (bottom part). 
25 . 7.

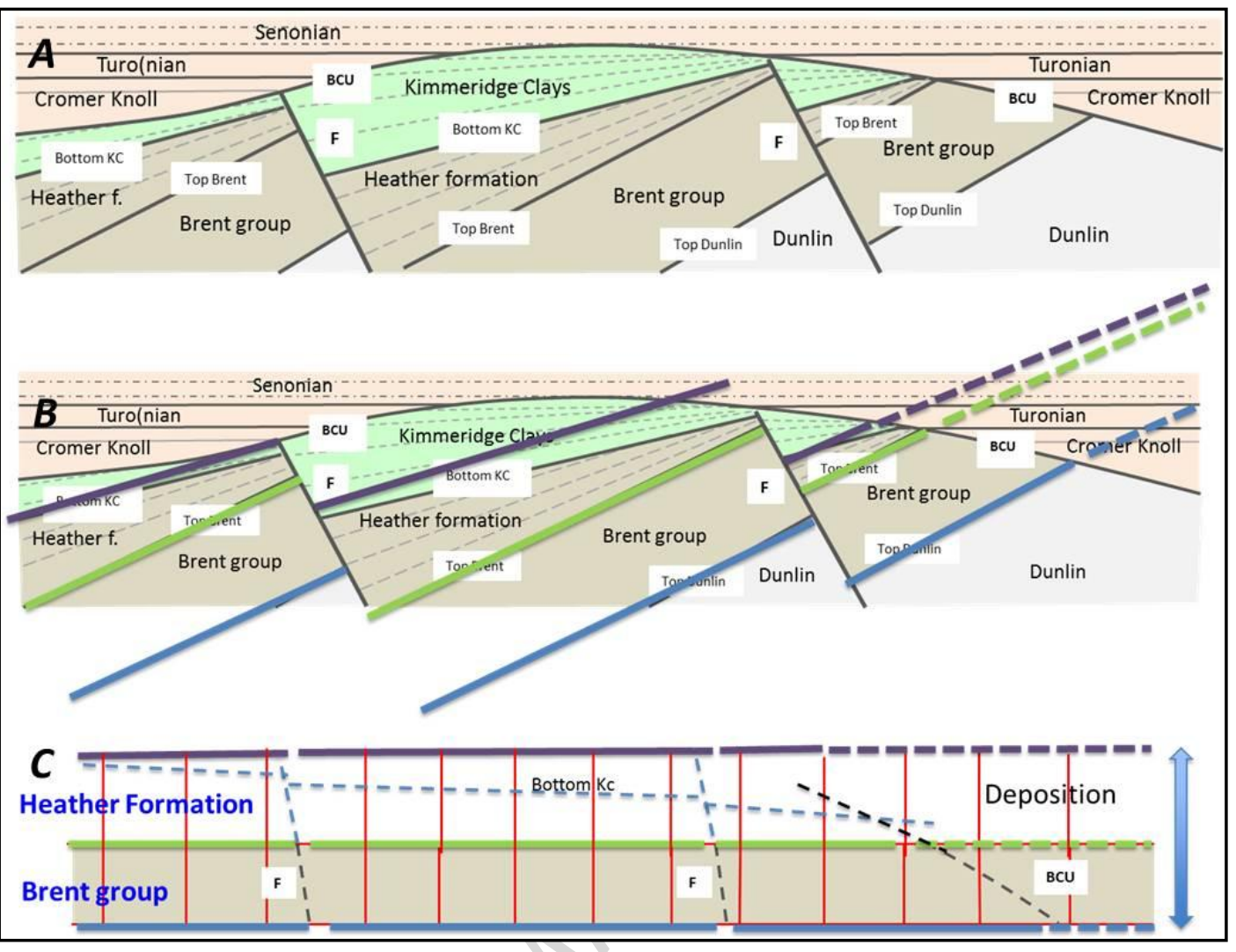

28. Figure 2: Schematic view inspired from North Sea Field (A) Present day Field structure on which Bottom KC and BCU are two successive erosive surfaces. (B) Top Dunlin, Top Brent and Top Heather extrapolations operated to retrieve their location before the Bottom KC and BCU erosive events. (C) Reconstitution (using surface flattening) of the relative locations (after the deposition event) of the Brent Group and the Top Heather "Chrono Tops" and "Chrono Bottoms" which are the geological surfaces to unfold. 
30.

32.

31.

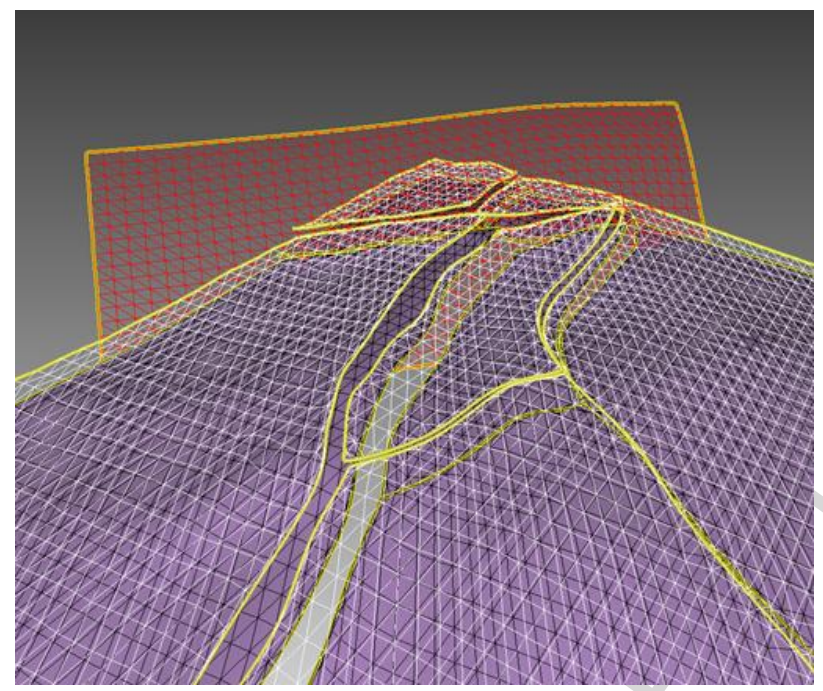

33. Figure 3: Example of micro- and macro-topology of the imported structural framework. 

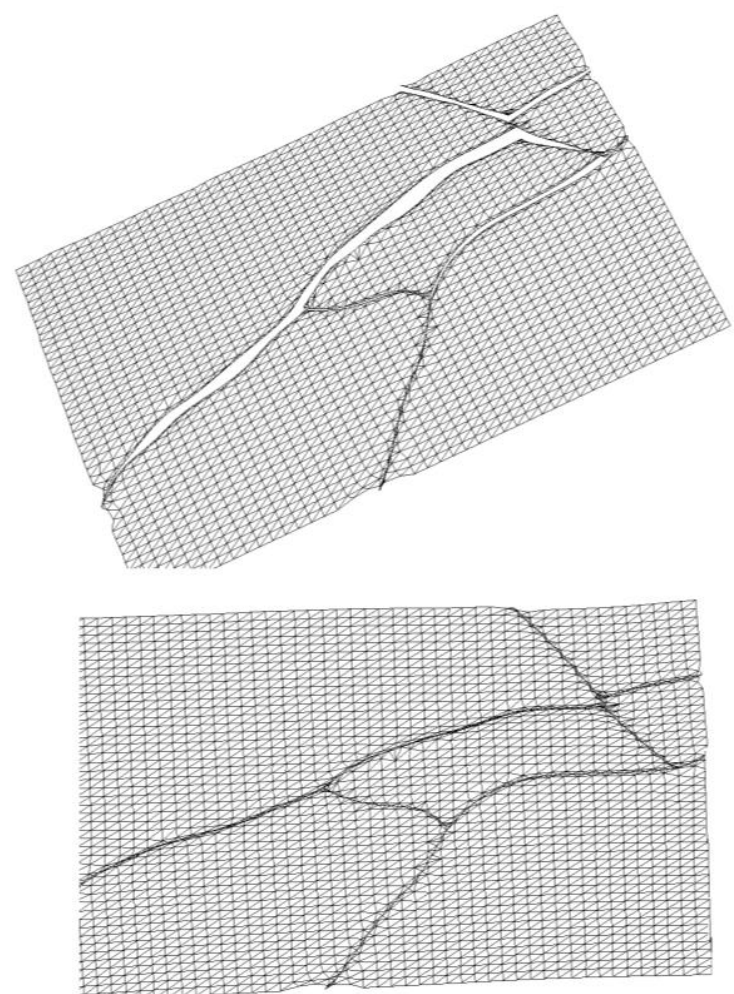

34.

35. Figure 4: TopBrent triangulated horizon to faults contacts before (up) and after flattening (botton).

36.

37.

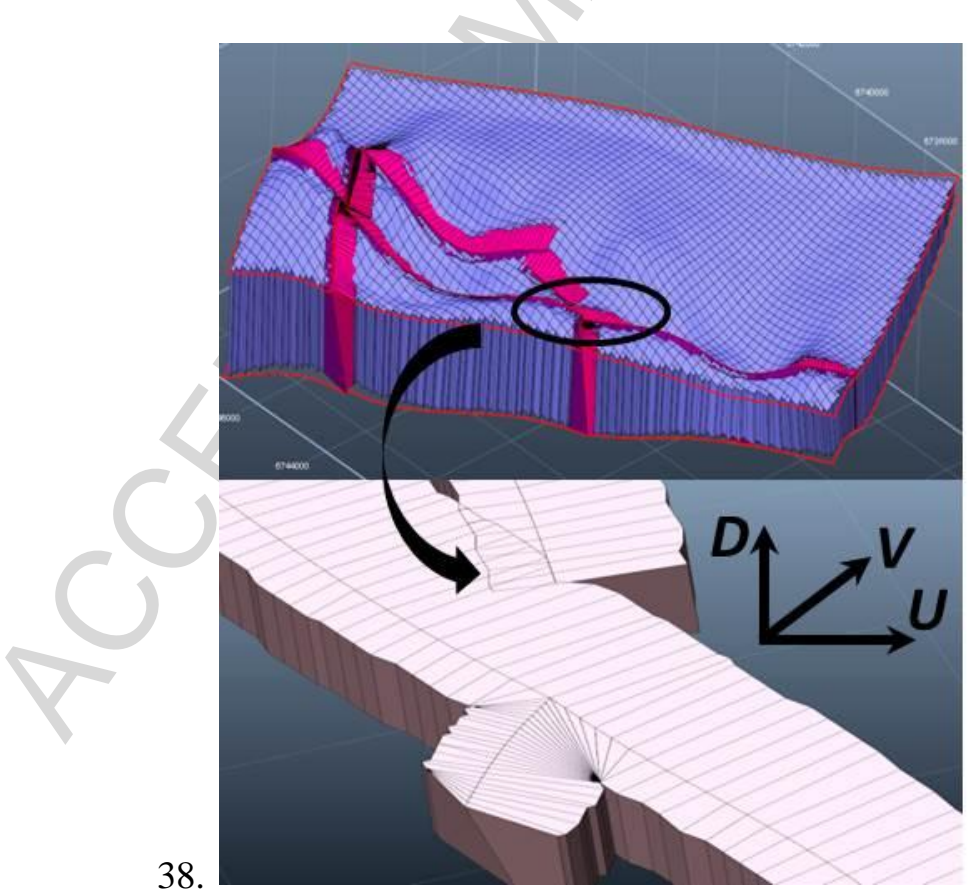

39. Figure 5: The thick Cartesian grid (up) containing a geological unit in the depositional $(U, V, D)$ domain. A zoomed area of the hexahedron mesh along part of the fault network (bottom). $U$ and $V$ are horizontal parametric coordinates and $D$ is the geological age. One can notice that the two hexahedron meshes are not coincident around the fault network. 
41.

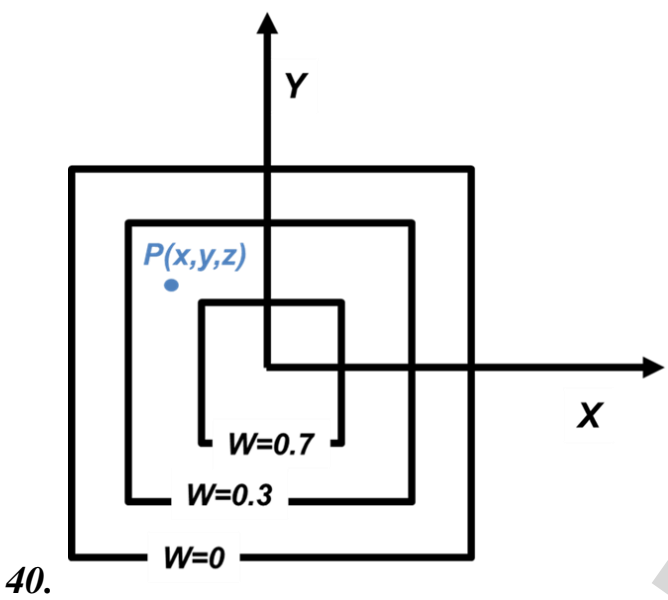

42. Figure 6: Example of 2D shape function used for localization. 3D shape functions are used (Dhatt and Touzot, 1981). A given point $P$ in a (X,Y,TWT or Depth) hexahedron has the same parametric coordinates in the transformed hexahedron in $(U, V, D)$ domain. This allows to transfer a property along a well from $(X, Y, T W T$ or Depth) to $(U, V, D)$ and after extrapolation in $(U, V, D)$ along geological time lines, transfer it back to $(X, Y, T W T$ or Depth) by using Finite Element Method (FEM) Shape functions. To compute a property in the transition area between structured and not structured meshes, and decide which one to take we select the cell in which the point has the heavier weight W, with : Weight = Min [ (1-x), (1-y), (1-z) ]. W characterizes the proximity between $P$ and the barycenter of the hexahedron. 

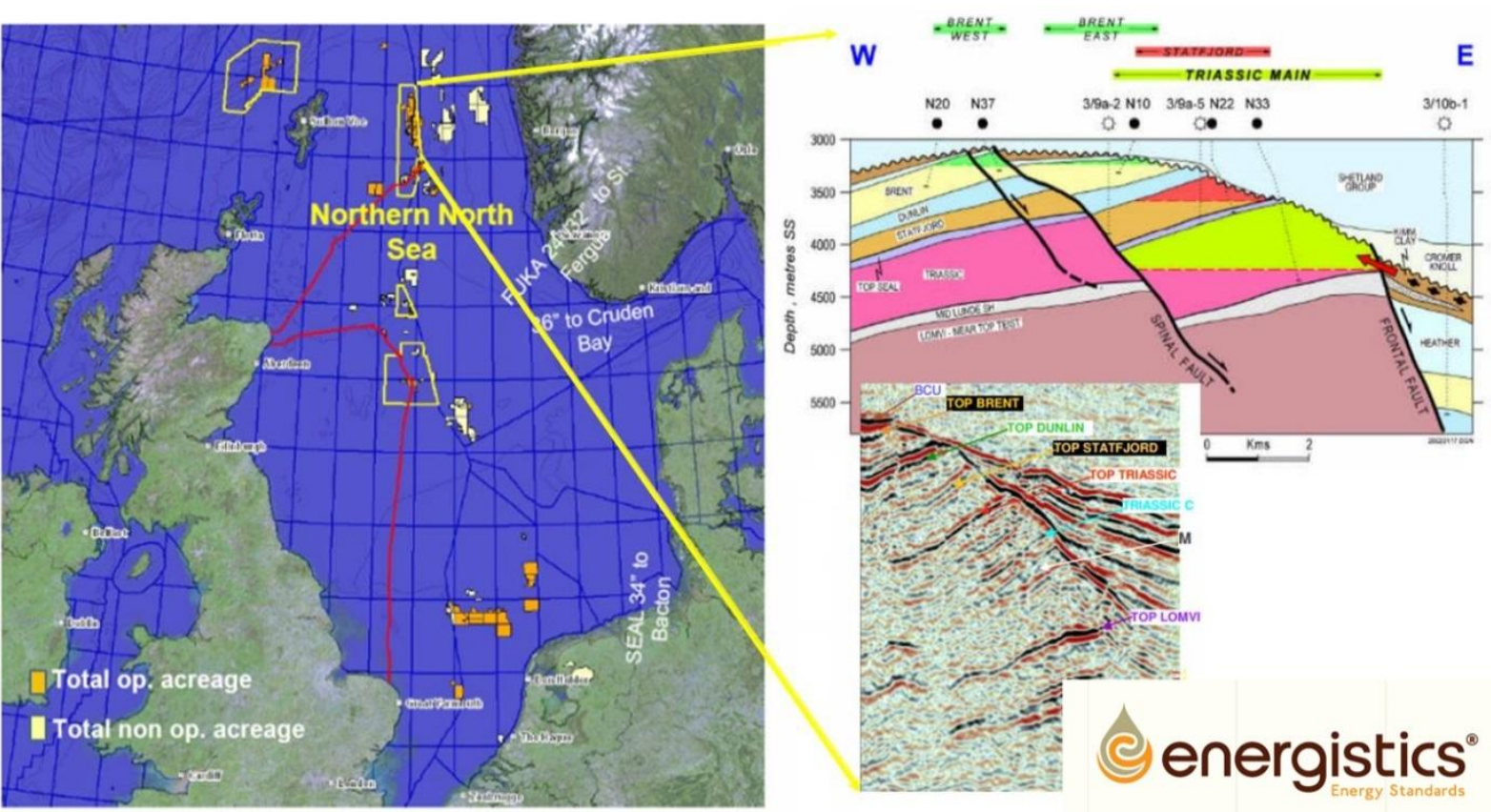

43.

44. Figure 7: The North Sea field (former campaign provided by TOTAL to exchange information and test methods): location map (left), Cross-section (right) extracted from Morandini et al. (2011). 


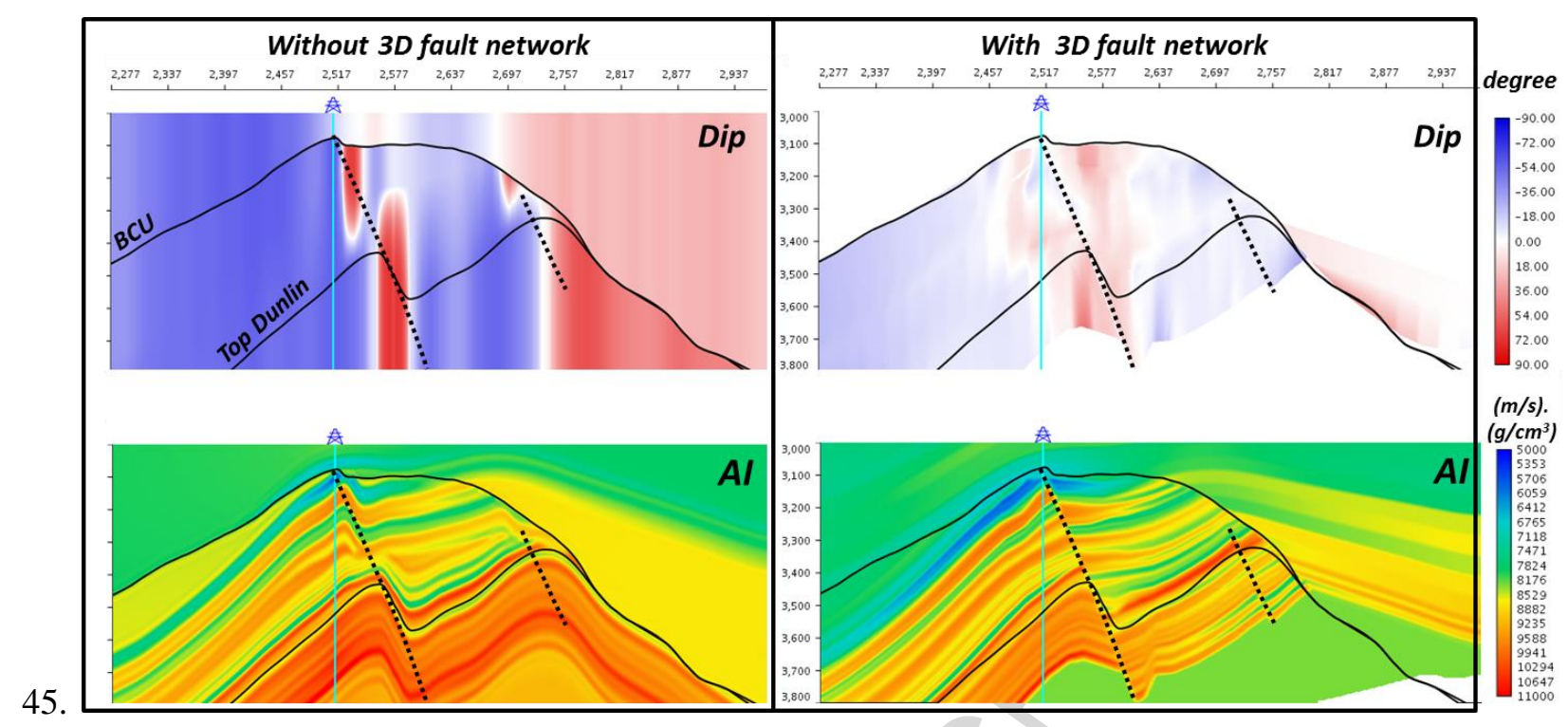

46.

47. Figure 8: InLine 2322 Dip associated to the non faulted a priori model (top left) and the faulted one (top right). The dips of the stratification is respected between the two sides of the faults (black dashed lines). Impedance from the non faulted a priori model (bottom left) and from the faulted model (bottom right). 
48.
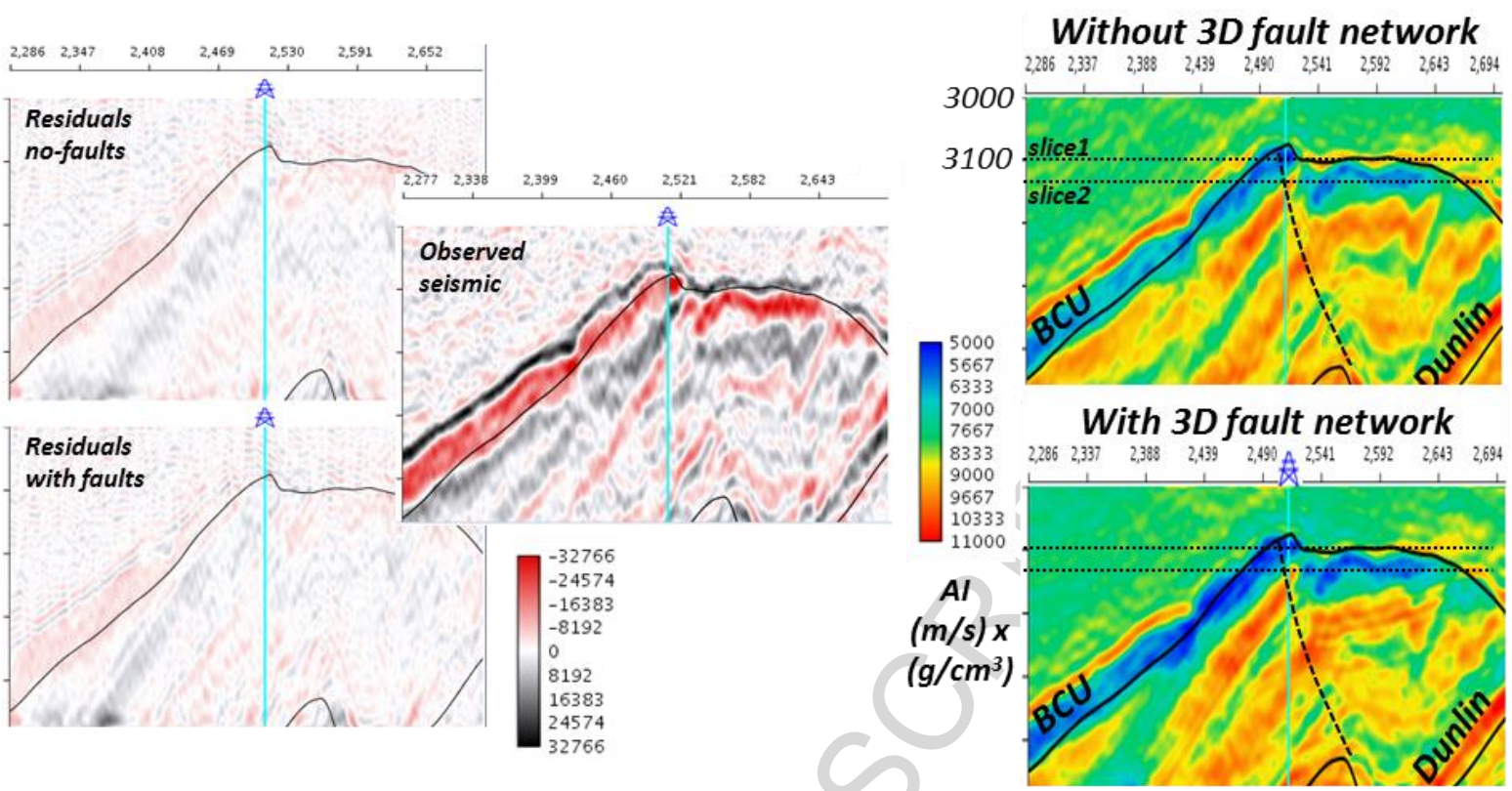

49.

50. Figure 9: Comparison of residuals (left) with the observed seismic (middle). Comparison of inverted Acoustic Impedance using a non faulted a priori model (top right) and a faulted one (bottom right).

51.

53. Figure 10: Inverted impedance using non faulted a priori model $(\mathrm{A}, \mathrm{C})$ and using a
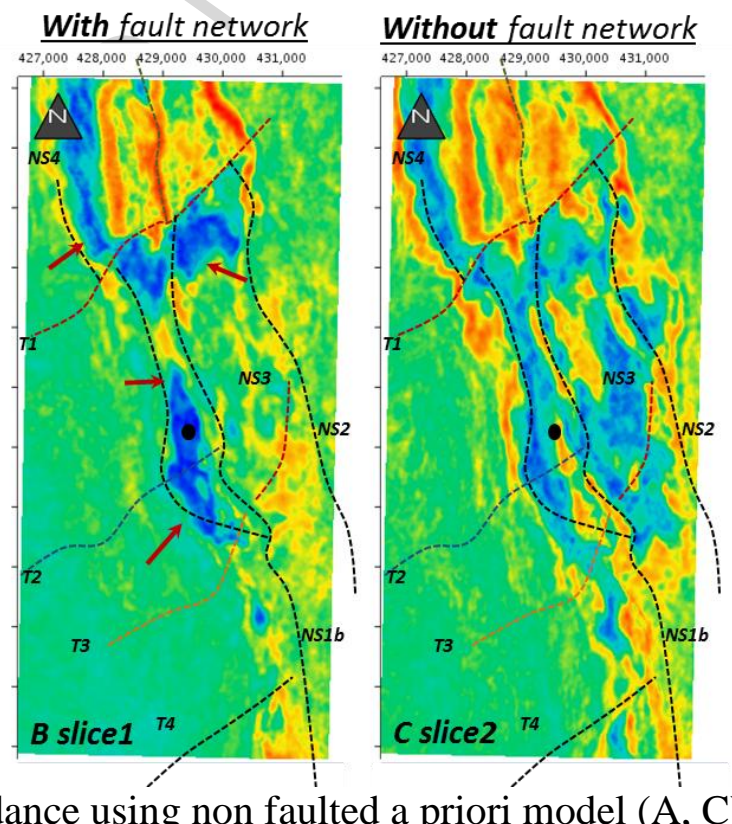

With fault network
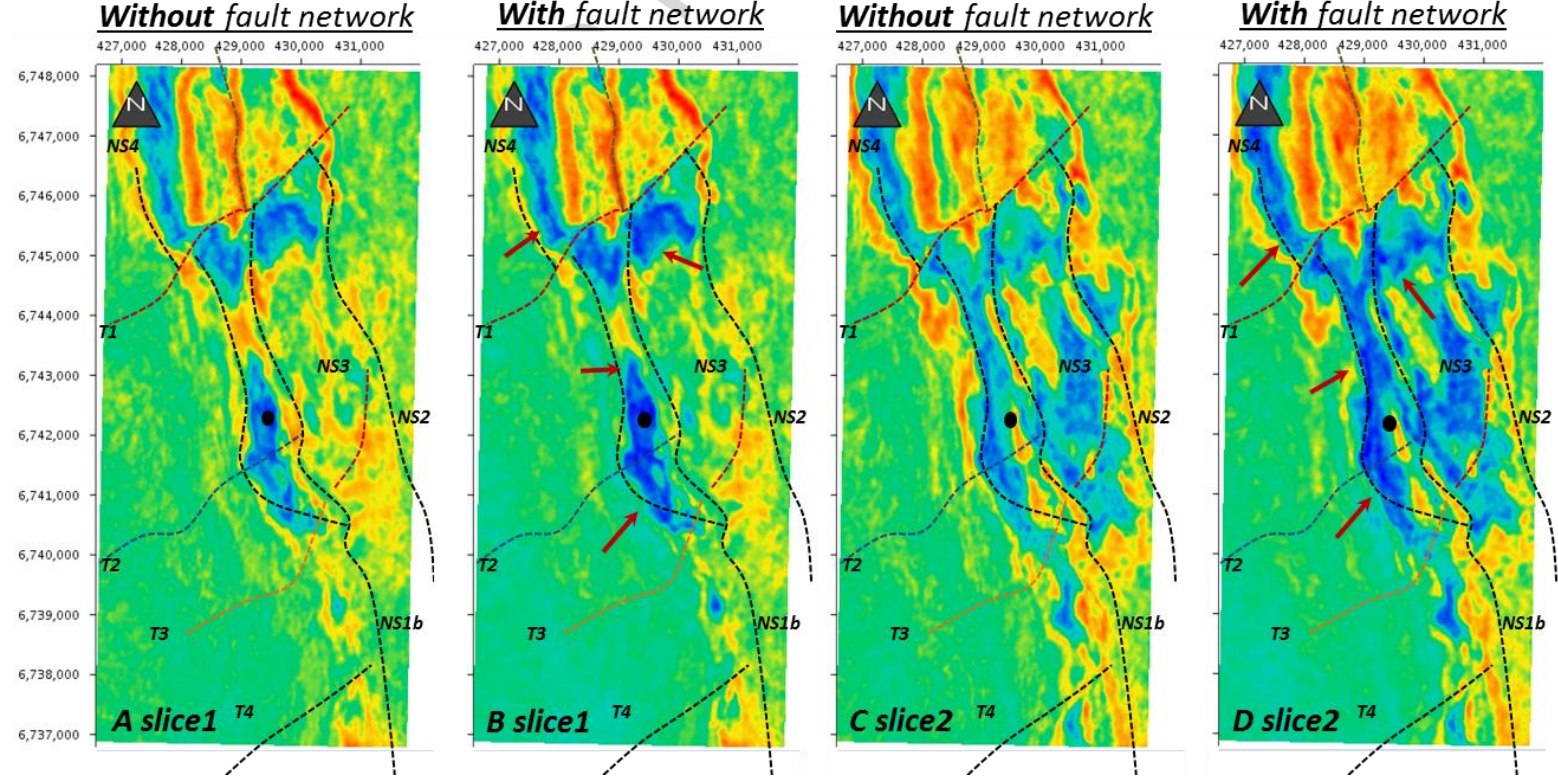

faulted one (B, D). One can note a better delineation of the Top Brent (in blue) obtained by inversion using the faulted a priori model (B, D). Slices A, B are at 3100 and slices C, $\mathrm{D}$ are at 3130 meters depth (see figure 9). 
Highlights

- We present a new way of integrating a 3D fault network in the earth parameter modeling and stratigraphic inversion workflow.

- We compared stratigraphic inversion results from a real case study, the UK Northern North Sea field, with and without integrating 3D fault network.

- Integration of 3D fault network at seismic scale is of high interest for reservoir characterization. 